\section{ATIVIDADE FÍSICA E ESTADO NUTRICIONAL: FATOR DE PROTEÇÃO PARA DOENÇAS CRÔNICAS NÃO TRANSMISSÍVEIS (DCNT) EM IDOSAS?}

\author{
Physical activity and nutritional status influence in the rise of \\ chronic noncommunicable diseases in old women? \\ Actividad física y el estado nutricional: factor de protección \\ para las enfermedades crónicas no transmisibles (ECNT) en \\ mujeres mayores?
}

\section{RESUMO}

Objetivo: Analisar a relação da prática de atividade física e do estado nutricional com as doenças crônicas não transmissíveis (DCNT) em mulheres idosas. Métodos: Estudo observacional, analítico, transversal, amostra por conveniência, amostra de 367 mulheres idosas (60 anos ou mais) das cidades de Presidente Prudente-SP e Uberaba-MG, entre outubro/2010 e agosto/2012. Para identificação das DCNT, utilizou-se um questionário baseado no Standard Health Questionnaire (SHQ), o Questionário Internacional de Atividade Física (IPAQ), para avaliar o nível de atividade física e as variáveis antropométricas para o estado nutricional. $\mathrm{Na}$ análise estatística, utilizou-se o teste qui-quadrado para verificar a associação entre o nível de atividade física, segundo estado nutricional e presença de doenças cardiometabólicas, e regressão logística binária para testar a magnitude dessas associações. Resultados: Houve fator de proteção contra a hipertensão para as mulheres consideradas eutróficas ativas $(\mathrm{p}=0,024)$ e eutróficas sedentárias $(\mathrm{p}=0,032)$ quando comparadas ao grupo de risco (sedentárias e com sobrepeso/obesidade), porém o mesmo não foi observado nas com excesso de peso e consideradas ativas fisicamente $(p=0,734)$. Houve associação positiva entre as idosas eutróficas sedentárias $(\mathrm{p}=0,047)$ em relação ao risco para colesterol. Quando observado, o diabetes não obteve associação em nenhum dos grupos. Conclusão: A atividade física não foi considerada como fator de proteção para hipercolesterolemia, diabetes e hipertensão, possivelmente por causa da causalidade reversa, pois, a partir do diagnóstico da doença, essas mulheres, antes sedentárias, iniciaram a prática de atividade física, contribuindo para esses resultados.

Descritores: Atividade Física; Doença Crônica; Saúde da Mulher; Idoso.

\section{ABSTRACT}

Objective: To analyze the association of regular physical activity and nutritional status with chronic noncommunicable diseases (NCDs) in elderly women. Methods: Observational and analytical cross-sectional study conducted with a convenience sample of 367 elderly women (60 and over) from the cities of Presidente Prudente, SP and Uberaba, MG, between October/2010 and August/2012. NCDs were identified using a questionnaire based on the Standard Health Questionnaire (SHQ). The International Physical Activity Questionnaire (IPAQ) was used to assess the level of physical activity and anthropometric variables were used to assess the nutritional status. Statistical analysis included the chi-squared test to check for association between physical activity level, according to nutritional status, and the presence of cardiometabolic diseases, and binary logistic regression to test the magnitude of these associations. Results: There was a protective factor against hypertension in active eutrophic women $(p=0.024)$ and sedentary eutrophic women $(p=0.032)$ when compared to the risk group (sedentary and overweight/obesity women); however, this was not observed in physically active overweight women $(p=0.734)$. There was a positive association between sedentary eutrophic elderly women $(p=0.047)$ and the risk for cholesterol. None of the groups were associated with diabetes. Conclusion: Physical activity was not considered a protective factor for hypercholesterolemia, diabetes and hypertension, possibly because of reverse causality given that sedentary women started physical activity after the diagnosis of the disease, which contributed to these results.

Descriptors: Physical activity; Chronic Disease; Women's Health. Elderly.
Artigo Original

1) Universidade Federal do Amazonas UFAM - Parintins (AM) - Brasil

2) Escola de Saúde Pública da Universidade de São Paulo - ESP/USP - São Paulo (SP) - Brasil

3) Universidade Estadual Paulista - UNESP - Rio Claro (SP) - Brasil

4) Universidade Estadual Paulista - UNESP - Presidente Prudente (SP) - Brasil

5) Universidade Federal do Triângulo Mineiro - UFTM - Uberaba (MG) - Brasil

Recebido em: 25/11/2013 Revisado em: 11/03/2014 Aceito em: 12/09/2014 


\section{RESUMEN}

Objetivo: Analizar la relación de la práctica de actividad física y el estado nutricional con las enfermedades crónicas no transmisibles (ECNT) en mujeres mayores. Métodos: Estudio observacional, analítico, transversal de muestreo por conveniencia y muestra de 367 mujeres mayores (60 años o más) de las ciudades de Presidente Prudente-SP y Uberaba-MG, entre octubre/2010 y agosto/2012. Se utilizó un cuestionario basado en el Standard Health Questionnaire (SHQ) para la identificación de las ECNT, el Cuestionario Internacional de Actividad Física (IPAQ) para evaluar el nivel de actividad física y las variables antropométricas para evaluar el estado nutricional. En el análisis estadístico se utilizó la prueba de Chi-cuadrado para verificar la asociación del nivel de actividad física según el estado nutricional y la presencia de enfermedades cardiometabolicas y la regresión logística binaria para testar la magnitud de estas asociaciones. Resultados: Se encontró un factor de protección contra la hipertensión para las mujeres consideradas eutróficas activas $(p=0,024)$ y eutróficas sedentarias $(p=0,032)$ al comparar con el grupo de riesgo (sedentarias y con sobrepeso/obesidad), sin embargo lo mismo no se ha observado en aquellas con exceso de peso consideradas fisicamente activas $(p=0,734)$. Hubo asociación positiva entre las mujeres mayores eutróficas sedentarias $(p=0,047)$ y el riesgo de colesterol. No hubo asociación de la diabetes en ningún de los grupos. Conclusión: La actividad fisica no ha sido considerada como factor de protección para la hipercolesterolemia, la diabetes y la hipertensión, posiblemente debido la causalidad reversa, pues a partir del diagnóstico de la enfermedad estas mujeres, que antes eran sedentarias, empezaron la práctica de actividad fisica, contribuyendo con estos resultados.

Descriptores: Actividad Motora; Enfermedad Crónica; Salud de la mujer; Anciano.

\section{INTRODUÇÃO}

No Brasil, as doenças crônicas não transmissíveis (DCNT) compõem a principal causa de morte em adultos e idosos, com destaque para as doenças cardiovasculares e neoplasias. A presença dessas doenças, em sua maioria, está associada ao excesso de peso muito frequente entre as mulheres ${ }^{(1,2)}$ e indivíduos mais velhos, e são responsáveis pelo aumento dos gastos com os serviços, medicamentos e internações, prejudicando todo o sistema de saúde pública ${ }^{(3)}$.

A mais prevalente dessas DCNT na população adulta e idosa é a hipertensão arterial (HÁ), podendo chegar a valores superiores a $67 \%$ em idosos longevos ${ }^{(4)}$ e tendo relação direta com o desenvolvimento de outros agravos cardiovasculares $^{(5)}$. O diabetes, apesar de sua prevalência ser menor se comparada a outras DCNT, é uma doença altamente limitante, podendo causar cegueira, amputações, nefropatias, complicações cardiovasculares e encefálicas. A presença de dislipidemia também é preocupante, uma vez que o perfil lipídico desfavorável também pode aumentar o risco de complicações cardiovasculares ${ }^{(6)}$.

A prática de atividade física regular é considerada essencial para a promoção à saúde e o envelhecimento saudável, reduzindo o risco de DCNT e morte prematura, além de proporcionar importantes benefícios para a saúde fisiológica, funcional e mental ${ }^{(7,8)}$.

Portanto, mesmo sendo tema bastante explorado na literatura, são necessárias investigações que apresentem resultados sobre a eficácia da prática de atividade física segundo o estado nutricional para prevenção e tratamento de DCNT, especialmente em mulheres a partir dos 50 anos. Sendo assim, o objetivo deste estudo foi analisar a relação da prática de atividade física e do estado nutricional com as DCNT em mulheres idosas.

\section{MÉTODOS}

Trata-se de um estudo observacional, analítico, com delineamento transversal. A amostra selecionada por conveniência e composta por 367 mulheres idosas (60 anos ou mais), residentes na zona urbana de dois municípios participantes de programas de exercícios físicos, intitulados "Medicina Preventiva", oferecido por um plano de saúde privado da cidade de Presidente Prudente-SP, e "Ginástica Orientada", oferecido em locais públicos da cidade de Uberaba-MG, no período de outubro/2010 a agosto/2012.

As variáveis antropométricas coletadas foram peso corporal, estatura e circunferência de cintura (CC). O peso corporal foi aferido com a utilização de uma balança digital $\left(\right.$ Plenna $\left.^{\circledR}\right)$, com precisão de $0,1 \mathrm{~kg}$ e capacidade máxima de $150 \mathrm{~kg}$. A estatura foi aferida em um estadiômetro portátil (Sanny), com precisão de $0,1 \mathrm{~cm}$. Os valores obtidos de peso e estatura foram utilizados para o cálculo do índice de massa corporal (IMC) [peso( $\mathrm{kg}) /$ altura $\left.(\mathrm{m})^{2}\right]$. O ponto de corte adotado para indicar a presença de sobrepeso foi $\geq 27$ $\mathrm{kg} / \mathrm{m}^{2(9)}$. Para a avaliação da circunferência de cintura (CC), foi utilizada uma fita metálica com precisão de $0,1 \mathrm{~mm}$. Os procedimentos adotados na mensuração antropométrica foram realizados conforme descritos ${ }^{(10)}$.

Para identificar a prevalência de DCNT, aplicou-se o questionário baseado no Standard Health Questionnaire (SHQ), que analisa a presença de DCNT nas populações adulta e idosa. O questionário utiliza questões de dupla escolha (presença ou ausência de algumas doenças DCNT), distribuídas em três grupos: metabólicas, cardiovasculares e osteoarticulares. Para este estudo, foram adotadas as seguintes DCNT: (I) hipertensão arterial, (II) diabetes e (III) colesterol $^{(11)}$.

Para avaliar o nível de atividade física (NAF), utilizouse o Questionário Internacional de Atividade Física (IPAQ), versão 8 , na sua forma curta ${ }^{(12)}$. O IPAQ é um instrumento 
que permite estimar o tempo semanal de gasto na realização de atividades físicas de intensidade moderada e vigorosa e em diferentes contextos da vida (trabalho, tarefas domésticas, transporte e lazer). Para o presente estudo, as mulheres consideradas ativas realizavam 150 minutos ou mais de atividade moderada e/ou intensa por semana ${ }^{(13,14)}$.

As avaliações foram aplicadas individualmente por duas entrevistadoras e dois auxiliares treinados previamente para a aplicação do questionário e padronização da coleta.

Utilizou-se o software Epidata, versão 3.1b, e as análises por meio do pacote estatístico SPSS (versão 20.0). Para as variáveis numéricas, a normalidade do conjunto de dados foi confirmada pelo teste de Komolgorov-Smirnof (K-S). Assim, a estatística descritiva foi composta por valores de média e desvio padrão. As avaliadas foram distribuídas em dois grupos (ativas e sedentárias), e dentro de cada grupo foram feitos mais dois subgrupos. Então, foram comparados os valores médios de cada variável antropométrica e NAF, analisado por meio do teste $t$ de Student para amostras independentes.

A regressão logística binárias, representada por valores de razão de chance (RC), com intervalo de confiança de $95 \%$ (IC 95\%), indicou a magnitude das associações entre estado nutricional (eutrófica e sobrepeso/obesidade) e DCNT. Em todas as análises, adotou-se o nível de significância de 5\%.

Esta pesquisa seguiu os princípios éticos presentes na Declaração de Helsinque e na Resolução no 466/12 do Conselho Nacional de Saúde. Além disso, os protocolos foram revisados e aprovados pelo Comitê de Ética em Pesquisas da Universidade Estadual Paulista de Presidente Prudente (Processo n ${ }^{\circ}$ 298/2008).

\section{RESULTADOS}

Participaram do estudo 367 mulheres idosas. Destas, 295 (80,4\%) foram consideradas ativas e participaram - em média, 1 ano e 3 meses - de programas de atividade física.

A Tabela I apresenta os valores descritivos de média e desvio padrão das variáveis numéricas estudadas e a comparação dentro de cada grupo (sedentária e ativa). No grupo sedentário, as variáveis idade, peso, estatura, IMC e CC apresentaram diferença $(p=0,001)$ entre os subgrupos eutróficas e sobrepeso/obesidade. Já no grupo de mulheres ativas, quando os subgrupos foram comparados, pôde-se observar que apenas as variáveis antropométricas apresentaram diferenças significativas $(\mathrm{p}=0,001)$.

Tabela I - Características gerais da amostra, com média e desvio padrão, distribuída entre os diferentes grupos e subgrupos de mulheres idosas. Presidente Prudente-SP e Uberaba-MG, 2012.

\begin{tabular}{|c|c|c|c|c|c|c|c|c|}
\hline \multirow{3}{*}{ Variáveis } & \multicolumn{2}{|c|}{ Sedentárias } & \multirow[b]{3}{*}{$\mathbf{t}$} & \multirow[b]{3}{*}{$p$} & \multicolumn{2}{|c|}{ Ativas } & \multirow[b]{3}{*}{$\mathbf{t}$} & \multirow[b]{3}{*}{$p$} \\
\hline & $\begin{array}{l}\text { Eutróficas } \\
\quad(n=42)\end{array}$ & $\begin{array}{c}\text { Sobrepeso } \\
\text { e obesidade } \\
(\mathrm{n}=\mathbf{3 0})\end{array}$ & & & $\begin{array}{c}\text { Eutróficas } \\
(n=143)\end{array}$ & $\begin{array}{c}\text { Sobrepeso } \\
\text { e obesidade } \\
(n=152)\end{array}$ & & \\
\hline & \multicolumn{2}{|c|}{ Média (DP) } & & & \multicolumn{2}{|c|}{ Média (DP) } & & \\
\hline Idade (anos) & $79,9(7,0)$ & $75,5(9,7)$ & 2,28 & $0,026^{*}$ & $68,7(6,5)$ & $67,3(6,3)$ & 1,84 & 0,067 \\
\hline Peso $(\mathrm{kg})$ & $53,4(7,6)$ & $71,9(7,1)$ & $-10,39$ & $0,001^{*}$ & $57,1(6,9)$ & $74,8(10,4)$ & $-17,20$ & $0,001 *$ \\
\hline Estatura $(\mathrm{cm})$ & $151,2(7,1)$ & $153,3(6,2)$ & $-1,34$ & 0,182 & $154,6(6,0)$ & $155,2(6,5)$ & $-7,47$ & 0,456 \\
\hline IMC $(\mathrm{kg} / \mathrm{m} 2)$ & $23,3(2,5)$ & $30,6(2,6)$ & $-11,97$ & $0,001^{*}$ & $23,8(2,3)$ & $31,0(3,6)$ & $-20,57$ & $0,001^{*}$ \\
\hline $\mathrm{CC}(\mathrm{cm})$ & $81,5(7,3)$ & $97,0(9,7)$ & $-7,74$ & $0,001^{*}$ & $81,1(8,2)$ & $95,9(9,1)$ & $-14,66$ & $0,001 *$ \\
\hline NAF (min) & $7,3(19,5)$ & $8,7(20,8)$ & $-2,94$ & 0,770 & $\begin{array}{c}349,6 \\
(463,8)\end{array}$ & $327,2(436,1)$ & 0,427 & 0,670 \\
\hline
\end{tabular}

DP: desvio padrão; IMC: índice de massa corporal; CC: circunferência de cintura; NAF: nível de atividade física. ${ }^{*} \mathrm{p}<0,05$

Tabela II - Associação entre nível de atividade física, segundo estado nutricional e hipertensão arterial em mulheres idosas. Presidente Prudente-SP e Uberaba-MG, 2012.

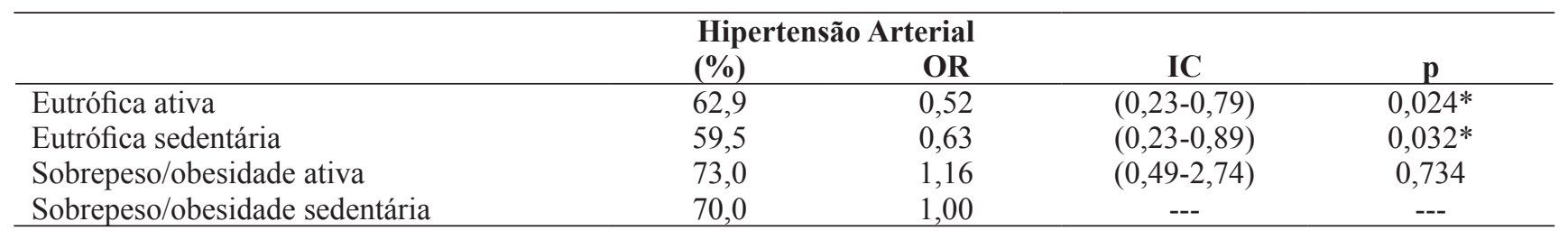

IC $=$ intervalo de confiança. ${ }^{*} \mathrm{p}<0,05$ 
Na Tabela II, são apresentadas as associações entre o estado nutricional segundo o nível de atividade física e hipertensão arterial em mulheres idosas, por meio da razão de chances. Houve fator de proteção para as mulheres eutróficas ativas $(\mathrm{p}=0,024)$ e eutróficas sedentárias ( $\mathrm{p}=0,032)$ comparadas ao grupo de risco sobrepeso/ obesidade. Isso não foi observado para aquelas com excesso de peso e ativas fisicamente $(\mathrm{p}=0,734)$.

Segundo o estado nutricional e colesterol, não houve associação quando se observou as mulheres eutróficas ativas $(\mathrm{p}=0,581)$, quando associadas ao grupo de risco constituído por aquelas mulheres consideradas sedentárias, com sobrepeso ou obesidade. Porém, houve associação positiva entre as idosas eutróficas sedentárias $(p=0,047)$ em relação à ocorrência de dislipidemia (Tabela III).

A Tabela IV mostra que não houve associação com o diabetes entre as mulheres ativas e eutróficas $(p=0,775)$, sedentárias e eutróficas $(\mathrm{p}=0,857)$, ativas e com excesso de peso $(\mathrm{p}=0,734)$.

Tabela III - Associação entre nível de atividade física estratificada, segundo estado nutricional e colesterol em mulheres idosas. Presidente Prudente-SP e Uberaba-MG, 2012.

\begin{tabular}{|c|c|c|c|c|}
\hline \multicolumn{5}{|c|}{ Colesterol } \\
\hline & $(\%)$ & OR & IC & $p$ \\
\hline Eutrófica ativa & 31,5 & 0,79 & $(0,79-1,81)$ & 0,581 \\
\hline Eutrófica sedentária & 16,7 & 0,35 & $(0,12-0,92)$ & $0,047 *$ \\
\hline Sobrepeso/obesidade ativa & 38,8 & 1,10 & $(0,49-2,47)$ & 0,825 \\
\hline Sobrepeso/obesidade sedentária & 36,7 & 1.00 & --- & --- \\
\hline
\end{tabular}

$\mathrm{IC}=$ intervalo de confiança; $\mathrm{OR}=$ Odds ratio. ${ }^{*} \mathrm{p}<0,05$

Tabela IV - Associação entre nível de atividade física estratificada, segundo estado nutricional e diabetes em mulheres idosas. Presidente Prudente-SP e Uberaba-MG, 2012.

\begin{tabular}{lcccc}
\hline & \multicolumn{2}{c}{ Diabetes } & & \\
& $\mathbf{( \% )}$ & OR & IC & $\boldsymbol{p}$ \\
\hline Eutrófica ativa & 15,4 & 0,78 & $(0,38-3,72)$ & 0,775 \\
Eutrófica sedentária & 11,9 & 0,86 & $(0,22-3,59)$ & 0,857 \\
Sobrepeso/obesidade ativa & 15,8 & 1,22 & $(0,39-3,81)$ & 0,734 \\
Sobrepeso/obesidade sedentária & 13,3 & 1,00 & --- & -- \\
\hline
\end{tabular}

IC $=$ intervalo de confiança; $\mathrm{OR}=$ Odds ratio. ${ }^{*} \mathrm{p}<0,05$

\section{DISCUSSÃO}

O presente estudo apresentou uma amostra com $80,4 \%$ das participantes ativas fisicamente. Esse resultado é positivo e relevante, uma vez que estudos prévios relatam o engajamento mínimo da população brasileira em atividade física, em que cerca de $40-45 \%$ não atingem as recomendações quanto à prática de atividade física, e esse número cresce precisamente quando especificadas apenas as atividades realizadas no período de lazer, encontrandose prevalências bem maiores, que variam de $65 \%$ a $97 \%{ }^{(15)}$. Essa diferença pode vincular-se à amostra por conveniência, ou seja, uma parcela específica da população que é fisicamente ativa.

Verificou-se também um aspecto bastante importante e que merece atenção: 295 mulheres da amostra, além de serem ativas, também apresentaram excesso de peso, ou seja, mais de $50 \%$ da amostra tem chances de desenvolver doenças crônicas não transmissíveis, pois esse perfil é considerado fator de risco independente ${ }^{(16)}$. Estudos na literatura evidenciam que indivíduos com excesso de peso, principalmente com obesidade abdominal, estão mais predispostos a apresentar DCNT, com influências negativas para a aptidão cardiorrespiratória e com maior risco de morbidade e mortalidade quando não tratadas ${ }^{(9,11)}$.

Entre as reações fisiológicas provocadas pelo excesso de peso, estão a sintetização da angiotensina II pelo tecido adiposo e alojamento em sua membrana da subunidade AT1 do receptor da angiotensina, com probabilidades de os componentes do sistema renina-angiotensina dos adipócitos compartilharem da fisiopatologia da hipertensão e doença cardiovascular. Além disso, o tecido adiposo sintetiza e secreta vários outros mediadores e citocinas próinflamatórias, que participam de mecanismos que induzem a dislipidemia, resistência à insulina, hipertensão arterial e aterosclerose ${ }^{(17)}$.

Os achados da presente pesquisa indicaram que mesmo o grupo das mulheres com excesso de peso consideradas ativas fisicamente não teve proteção contra a HA. Esse fato pode se dar devido à complexa relação e associação entre 
excesso de peso e hipertensão artéria, atribuída a fatores hemodinâmicos, como a resistência à insulina, a disfunção endotelial, o acúmulo de tecido adiposo, a ativação aumentada do sistema renina-angiotensina-aldosterona e do sistema nervoso simpático, ao lado do sistema endocanabinoide ${ }^{(18,19)}$.

A atividade física tem efeito agudo para determinados casos, porém, no que diz respeito aos aspectos fisiológicos, em especial à redução dos níveis pressóricos, para resultados positivos, é necessária atenção na intensidade, duração e frequência, com a prática de exercícios aeróbios - ou seja, mudança de comportamento ${ }^{(20)}$.

Quando se associou o nível de atividade física, as mulheres do presente estudo com excesso de peso, mesmo sendo consideradas ativas fisicamente, também não tiveram proteção. $\mathrm{O}$ excesso de peso, especialmente a obesidade abdominal, correlacionou-se com a maioria dos fatores de risco cardiovasculares, principalmente com níveis elevados de triglicérides e reduzidos de High Density Lipoproteins (HDL), apresentando maior impacto sobre a elevação da pressão arterial e consequentes danos para a saúde.

A literatura expõe com ostentação que o excesso de peso é considerado um dos fatores de alto risco para o desenvolvimento de doenças cardiovasculares, entre elas, a hipertensão arterial, com mais prevalência na população mais velha, aumentando com taxas exorbitantes ${ }^{(21)}$. Em contrapartida, um estudo apresentou que indivíduos com alta aptidão física, mesmo com excesso de peso, tiveram riscos reduzidos de morte de doença cardíaca, independentemente do estado de outros fatores de risco para doenças crônicas ${ }^{(22)}$.

Outro estudo epidemiológico com adultos fisicamente ativos apresentou em suas análises que o comportamento sedentário, em especial a quantidade de tempo gasto assistindo à televisão, é um preditor independente para as doenças metabólicas, diabetes e obesidade ${ }^{(7)}$.

Outro estudo caso-controle que objetivou determinar a contribuição da atividade física e obesidade abdominal à variação em biomarcadores inflamatórios para doenças relacionadas ao coração concluiu que participantes inativos, com uma circunferência de cintura elevada, foram caracterizados por níveis de deterioração dos marcadores inflamatórios, porém, vários marcadores foram associados com um risco aumentado de doenças cardíacas, independentemente da circunferência da cintura e dos níveis de atividade física ${ }^{(23)}$.

Além de ser fator de risco cardiovascular independente, a obesidade associa-se a uma série de outros fatores, como dislipidemia, hipercolesterolemia, hipertensão arterial e diabetes. Neste estudo, verificou-se que são necessárias mudanças no estilo de vida, mas com responsabilidade por parte do indivíduo. A maioria das mulheres da amostra apresentou comportamento fisicamente ativo, porém, não teve proteção às doenças crônicas associadas.
Há forte evidência científica de que a atividade física regular reduz o risco para o desenvolvimento e o agravamento da hipertensão arterial, da obesidade, do diabetes, da hipercolesterolemia e outras doenças crônicas não transmissíveis em adultos e idosos. No entanto, são necessárias pesquisas diversificadas para abordar importantes lacunas o conhecimento, incluindo a atenção ao tempo de comportamento sedentário ${ }^{(24,25)}$.

Apesar de todas essas hipóteses levantadas, é necessário considerar algumas limitações do estudo. O IMC para pessoas muito ativas ou ativas pode não ser um bom indicador. $\mathrm{O}$ fato de não ter sido considerado o consumo alimentar, o histórico familiar e os exames bioquímicos pode aumentar possíveis fatores de confusão.

Além disso, os estudos transversais dificultam o avanço em análises temporais dos fatores estudados, em virtude do viés de causalidade reversa, não permitindo encontrar relação de causalidade e estando passível de sofrer influências de fatores culturais e sociais. Assim, fazse necessária a realização de estudos de coorte para testar as hipóteses levantadas neste estudo.

\section{CONCLUSÃO}

A atividade física não foi considerada como fator de proteção para hipercolesterolemia, diabetes e hipertensão, possivelmente por causa da causalidade reversa, pois a partir do diagnóstico da doença, essas mulheres, antes sedentárias, iniciaram a prática de atividade física, contribuindo para esses resultados.

\section{AGRADECIMENTOS}

Agradecemos às participantes, à Cooperativa Médica Unimed da Cidade de Presidente Prudente, à Secretaria Municipal de Educação e Secretaria Municipal de Esporte e Lazer de Uberaba, e à Universidade Estadual Paulista de Presidente Prudente.

\section{REFERÊNCIAS}

1. Gallon CW, Wender MCO. Estado nutricional e qualidade de vida da mulher climatérica. Rev Bras Ginecol Obstet. 2012;34(4):175-83.

2. Veras RP. Envelhecimento populacional contemporâneo: demandas, desafios e inovações. Rev Saúde Pública. 2009;43(3):548-54.

3. Ministério da Saúde (BR). Diretrizes e recomendações para o cuidado integral de doenças crônicas não transmissíveis: promoção da saúde, vigilância, prevenção e assistência. Brasília: Ministério da Saúde; 2008. 
4. Gomes IC, Segatto AFM, Santos VR, Rosa CSC, Paulo TRS, Freitas Júnior IF. Aptidão Cardiorrespiratória e Envelhecimento como Indicadores de Risco de Obesidade. Rev Bras Cardiol. 2011;24(4):23-31.

5. Ferreira CCC, Peixoto MRG, Barbosa MA, Silveira EA. Prevalence of cardiovascular risk factors in elderly individuals treated in the brazilian Public Health System in Goiânia. Arq Bras Cardiol. 2010;95(5):621-8.

6. Regidor E, Franch J, Seguí M, Serrano R, RodríguezArtalejo F, Artola S. Traditional risk factors alone could not explain the excess mortality in patients with diabetes: a national cohort study of older Spanish adults. Diabetes Care. 2012;35(12):2503-9.

7. Healy GN, Dunstan DW, Salmon J, Shaw JE, Zimmet PZ, Owen N. Television time and continuous metabolic risk in physically active adults. Med Sci Sports Exerc. 2008;40(4):639-45.

8. Physical Activity Guidelines Advisory Committee. Physical Activity Guidelines Advisory Committee Report 2008. [acessed on July 15 2014]. Available at: http://www.health. gov/paguidelines/committeereport. aspx

9. Lipschitz DA. Screening for nutritional status in the elderly. Prim Care. 1994; 21(1):55-67.

10. Freitas Junior IF. Padronização de Técnicas Antropométricas. São Paulo: Cultura Acadêmica; 2009.

11. Castoldi RC, Moret DG, Gomes IC, Paulo TRS, Oikawa S, Freitas Júnior IF. Influência da adiposidade corporal sobre a aptidão cardiorrespiratória em mulheres idosas. Rev Bras Ciência Movimento. 2010;18(4)4:34-8.

12. Matsudo SM, Araújo TL, Matsudo VKR, Andrade DR, Andrade EL, Oliveira LC, Braggion G. Questionário Internacional de Atividade Física (IPAQ): estudo de validade e reprodutibilidade no Brasil. Rev Bras Atividade Física Saúde. 2001;6(2):5-18.

13. World Health Organization- WHO. Global recommendations on physical activity for health. WHO; 2010.

14. Haskell WL, Lee IM, Pate RR, et al. Physical activity and public health: updated recommendation for adults from the American College of Sports Medicine and the American Heart Association. Circulation. 2007;116(9):1081-93.

15. Hallal PC, Victora CG, Wells JC, Lima RC. Physical inactivity: prevalence and associated variables in Brazilian adults. Med Sci Sports Exerc. 2003;35(11):1894-900.
16. Ministério da Saúde (BR). Obesidade. Série Cadernos de Atenção Básica. Bras2009; 12:1-110. [acesso em 15 julho 2014]. Disponível em: http://nutricao.saude.gov. br/documentos/doc_obesidade.pdf.

17. Theodoro HMS, Rodrigues ADMS, Mendes KG, Liane RH, Paniz VMV, Olinto MTA. Reproductive characteristics and obesity in middle-aged women seen at an outpatient clinic in southern Brazil. Menopause. 2012;19(9):1022-8.

18. Jardim PCBV, Gondim MRSP, Monego ET, Moreira HG, Vitorino PVO, Souza WKSB, et al. Hipertensão arterial e alguns fatores de risco em uma capital brasileira. Arq Bras Cardiol. 2007;88(4):452-57.

19. Lobato NS, Akamine EH, Tostes RC, Carvalho MHC, Fortes ZB. Obesidade e hipertensão arterial. Rev Bras Hipertens. 2009;12(1)4-12.

20. Buchner DM. Physical activity and prevention of cardiovascular disease in older adults. Clin Geriatr Med. 2009;25(4):661-75.

21. Panagiotakos DB, Pitsavos C, Chrysohoou C, Skoumas I, Stefanadis C. Prevalence and five-year incidence (2001-2006) of cardiovascular disease risk factors in a Greek sample: the ATTICA study. Hellenic J Cardiol. 2009;50(5):388-95.

22. Sui X, Laditka JN, Hardin JW, Blair SN. Estimated functional capacity predicts mortality in older adults. J Am Geriatr Soc. 2007;55(12):1940-7.

23. Rana JS, Arsenault BJ, Despre's JP, Cote M, Talmud PJ, Ninio EJ, et al. Inflammatory biomarkers, physical activity, waist circumference, and risk of future coronary heart disease in healthymen and women. Eur Heart J. 2011;32(3):336-44.

24. Cornelissen VA, Fagard RH. Effects of endurance training on blood pressure, blood pressure regulating mechanisms, and cardiovascular risk factors. Hypertension. 2005; 46(4):667-75.

25. Lakerveld J, Dunstan D, Bot S, Salmon J, Dekker J, Nijpels G, et al. Abdominal obesity, TV-viewing time and prospective declines in physical activity. Prev Med. 2011; 53(4-5):299-302.

\section{Endereço para correspondência:}

Thais Reis Silva Paulo

Universidade Federal do Amazonas

Estrada do Macurany, $\mathrm{s} / \mathrm{n}$

Bairro: Jacareacanga

CEP: 69151000 - Parintins - AM - Brasil

E-mail: thais.reis.silva@hotmail.com 